\title{
A Circular Split Ringe Resonator (CSRR) Left Handed Metamaterial (LHM) having Simultaneous Negative Permeability and Permittivity
}

\author{
Moataza A. Hindy ${ }^{1}$, Ragab M. ElSagheer ${ }^{2}$ and M. S. Yasseen ${ }^{3 *}$ \\ ${ }^{1}$ Electronic Research Institute, Eltahrir st., Dokki, Cairo, Egypt \\ ${ }^{2}$ Electrical Engineering Department, Al-Azhar University, Nasr City, Cairo, Egypt \\ ${ }^{3}$ Electrical Engineering Department, Faculty of Engineering, \\ Al-Azhar University, Qena, Egypt

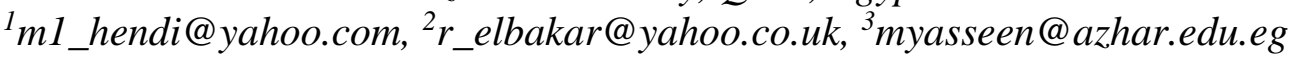

\begin{abstract}
This paper presents a design and simulation of a new structure having simultaneous negative permeability and permittivity which called double negative metamaterial (DNM) or left handed material (LHM). The LHM structure consists of five circular split ring resonators on one side of dielectric substrate and a set of wire couples on other side. CST Microwave Studio is used to model the LHM structure to calculate the scattering parameters (S11 and S21) from which the complex permittivity, permeability and refractive index can be extracted. MATLAB is used for verification of obtained negative values of structure's parameter. The circuit was manufactured and measured. The experimental results well agree with the simulated ones.
\end{abstract}

Keywords: Double negative material (DNM), left handed material (LHM), Negative Permeability, Negative Permittivity, Negative Refractive Index, Split Ring Resonators

\section{Introduction}

All materials can be described electrically by their complex permittivity $(\varepsilon)$ and permeability $(\mu)$ in frequency domain. These parameters can be determining the response of the material to electromagnetic (EM) radiation. The proper understanding of complex permittivity and permeability is required for all scientific and industrial applications. In manufacturing processes due to the unpredictability, the only way to find the parameters of materials is to measure them. For more than six decades the notion of metamaterials, that show negative permeability and permittivity, is drawn a lot of interest. These materials are also called left-handed materials (LHMs). (LHMs) is an artificial material having electromagnetic properties not found in nature. MTM have a lot of essential applications in communication circuits, science, technology and medicine. MTMs are first known by Russian scientist V.G. Veselago but the experimental verification did not occur until several decades [1]. Many recent papers have reveal the utility of metamaterials that lead to negative permeability, permittivity, and refractive indexes [2]-[8]. The University of California, San Diego group, which follow the work of Pendry et al. [3], [4], [9] in 2000 and demonstrated the first left-handed material [10], [11]. This Left-Handed Metamaterials (LHM) made use of nonmagnetic element, array of conducting SRR, and an array of conducting continuous wires to achieve a negative permittivity and negative permeability respectively. To realizing negative permeability, and permittivity, circular split ring resonators are used on the top side of a dielectric substrate FR4 while a couple of wires on back side. From this structure SRR gives negative permittivity, while the

\footnotetext{
* Corresponding Author
} 
wires in the back side give negative permeability, and negative refractive index is also carried out from the structure itself.

The LHM structure has been simulated in CST microwave studio 2012 software and scattering parameters (S11 and S21) are extracted to calculate negative values of permittivity, permeability and refractive index using direct retrieval method [12]-[14]. The LHM have a lot of applications in microwave engineering [15]. For LHM, we are interested in their response to electromagnetic fields. The most important application of LHM is to be used as a substrate or superstrate to improve the directivity and gain of antenna [16]-[18]. The other applications of LHMs are MTM-cloaking. The most attractive concept of LHM is the perfect lenses and near field imaging [15].

\section{Methodology}

The design of LHM structure combines five circular split ring resonators of copper having different radii $(\mathrm{R} 1-\mathrm{R} 5)$ but having the same width $\left(\mathrm{W}_{\mathrm{F}}\right)$ with spacing $(\mathrm{g})$ on one side of the substrate. Five rectangular wires of same length and width are printed on the other side of the substrate. The used substrate is FR4 having a dielectric constant of 4.3 and the thickness $1 \mathrm{~mm}$. The structure of LHM front view (Circular SRR) and back view (wires) are shown in Figure $1(\mathrm{a} \& \mathrm{~b})$ respectively.

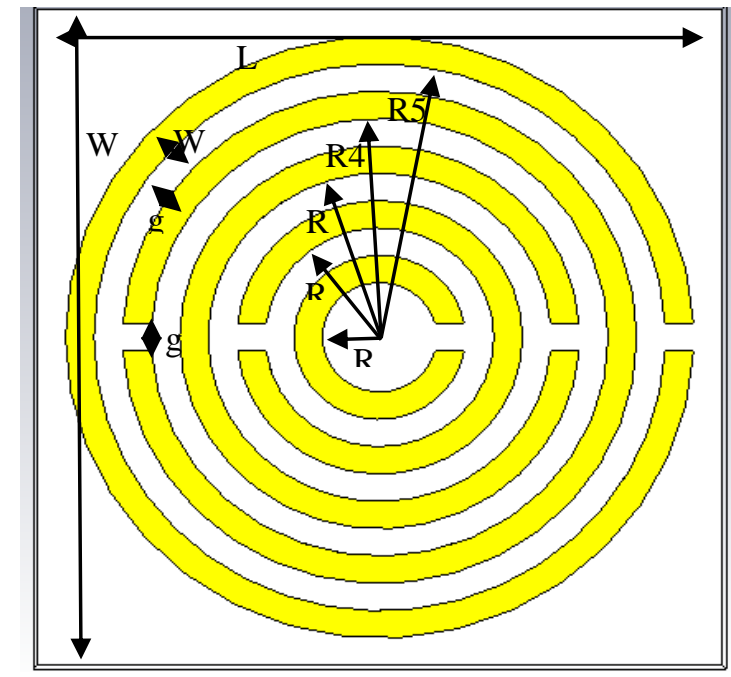

(a)

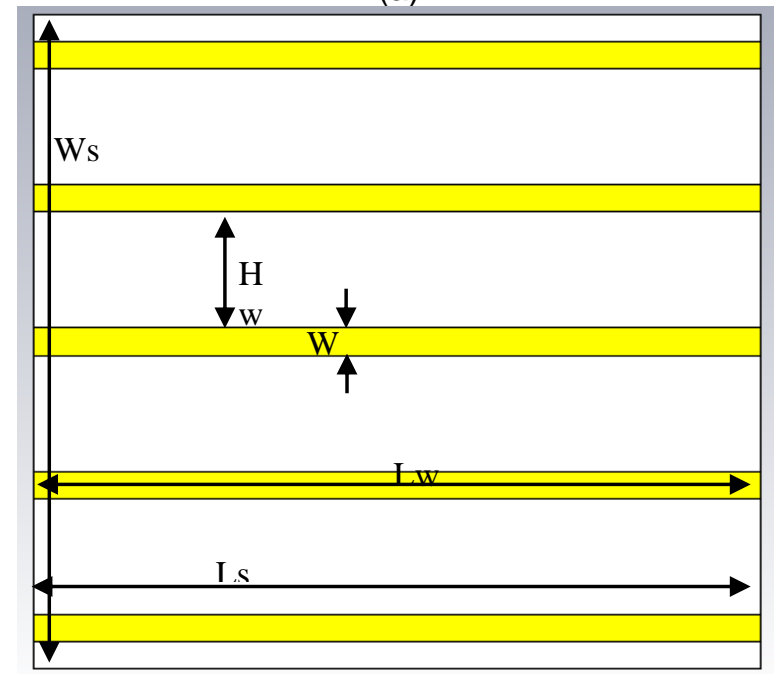

(b)

Figure 1. (A) Circular SRR Top View, (B) Wires Back View 
The parameters of circular split ring resonator, wires and substrate are summarized in Table 1. Various methods have been used to get negative permittivity and permeability from the simulated complex values of S-parameters. Nicolson-Ross-Weir (NRW) method is one of the extreme refined methods to find negative values of permittivity and permeability [19]-[22]. Other methods used Lorentz model and Drude model made some hypothesis about negative permittivity and permeability[14], [23].

\section{Table 1. The Parameters of Circular Split Ring Resonator}

\begin{tabular}{|c|c|c|}
\hline Parameters & Values & Unit \\
\hline Ls & 24 & $\mathrm{~mm}$ \\
\hline Ws & 24 & $\mathrm{~mm}$ \\
\hline R1 & 3 & $\mathrm{~mm}$ \\
\hline R2 & 5 & $\mathrm{~mm}$ \\
\hline R3 & 7 & $\mathrm{~mm}$ \\
\hline R4 & 9 & $\mathrm{~mm}$ \\
\hline R5 & 11 & $\mathrm{~mm}$ \\
\hline $\mathrm{Wr}$ & 1 & $\mathrm{~mm}$ \\
\hline $\mathrm{G}$ & 1 & $\mathrm{~mm}$ \\
\hline $\mathrm{Lw}$ & 22 & $\mathrm{~mm}$ \\
\hline $\mathrm{Ww}$ & 1 & $\mathrm{~mm}$ \\
\hline $\mathrm{Hw}$ & 4 & $\mathrm{~mm}$ \\
\hline
\end{tabular}

Between these and all other ways a direct retrieval method is used in this work which is more appropriate and straightforward[12].

The suggested LHM model is placed between two waveguide ports at the left and right of X-Axis. The wave is excited from positive $\mathrm{X}$-axis (Port 1) towards negative $\mathrm{X}$-axis (Port 2) to calculate complex S11 (reflection of port 1 at 1) and complex S21 (Transmission at port 2 due to 1) parameters. The Y-Plane is defined as a Perfect Electric Boundary (PEB) while Z-plane is defined as a Perfect Magnetic boundary (PMB). Simulated LHM metamaterial structure among two waveguide ports is shown in Figure (2).

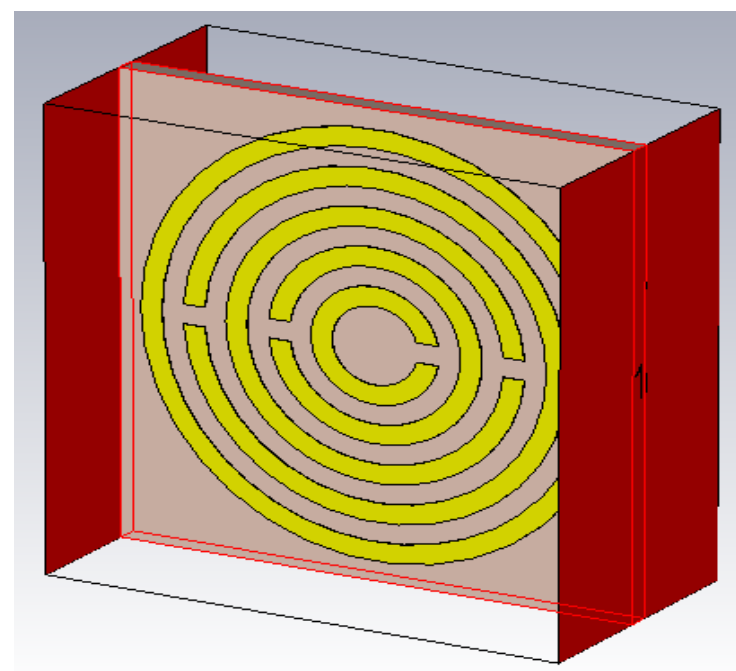

Figure 2. LHM Structure among Two Waveguide Ports

S-parameters can be obtained from corresponding structure using T/R Method by CST Microwave Studio. Exporting of scattering parameters (S11, and S21) to MATLAB one gets negative values of permittivity and permeability using the following equations [12]: 


$$
\mu=n z
$$

And

$$
\varepsilon=n / z
$$

Where

$$
n=\frac{1}{k d} \cos ^{-1}\left[\frac{1}{2 S_{21}}\left(1-S_{11}^{2}+S_{21}^{2}\right)\right]
$$

And

$$
z=\sqrt{\frac{\left(1+S_{11}\right)^{2}-S_{21}^{2}}{\left(1-S_{11}\right)^{2}-S_{21}^{2}}}
$$

\section{Simulated and Experimental Results Discussion}

Figure (3) shows the photographs of the improved fabricated Circular LHM cell. Top view shows the circular SRR, and the back view shows wires. Scattering parameters are the key terms for extracting negative permittivity and permeability. The complex scattering parameters S11, and S21 are extracted from CST Microwave studio. The simulation and measured scattering parameters S11, and S21 as depicted in Figure (4) shows good agreement. The structure resonates within the given range of frequency.

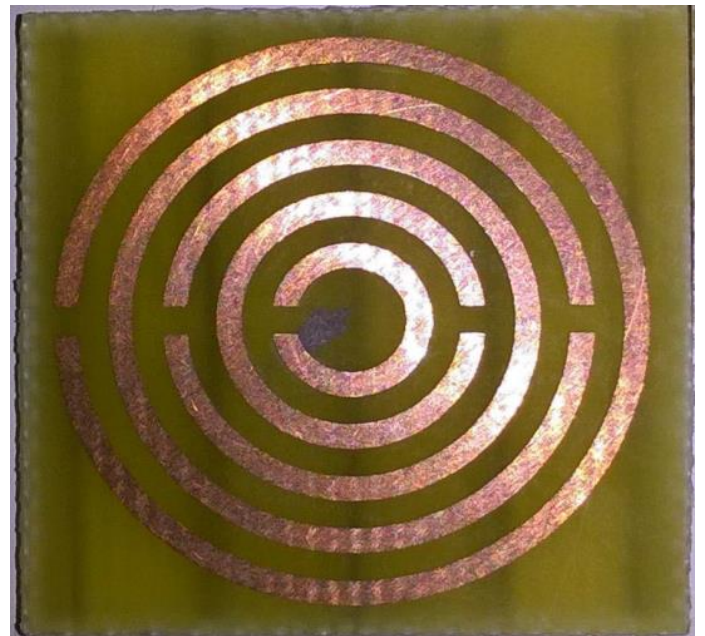

(a) Circular SRR Top View

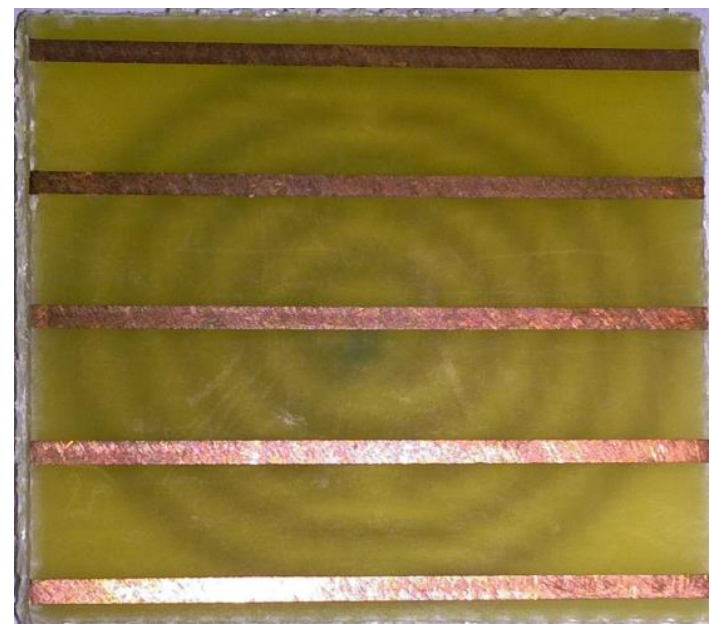

(b) Wires Back View

Figure 3. Photoghraphs of Fabricated LHM Cell 


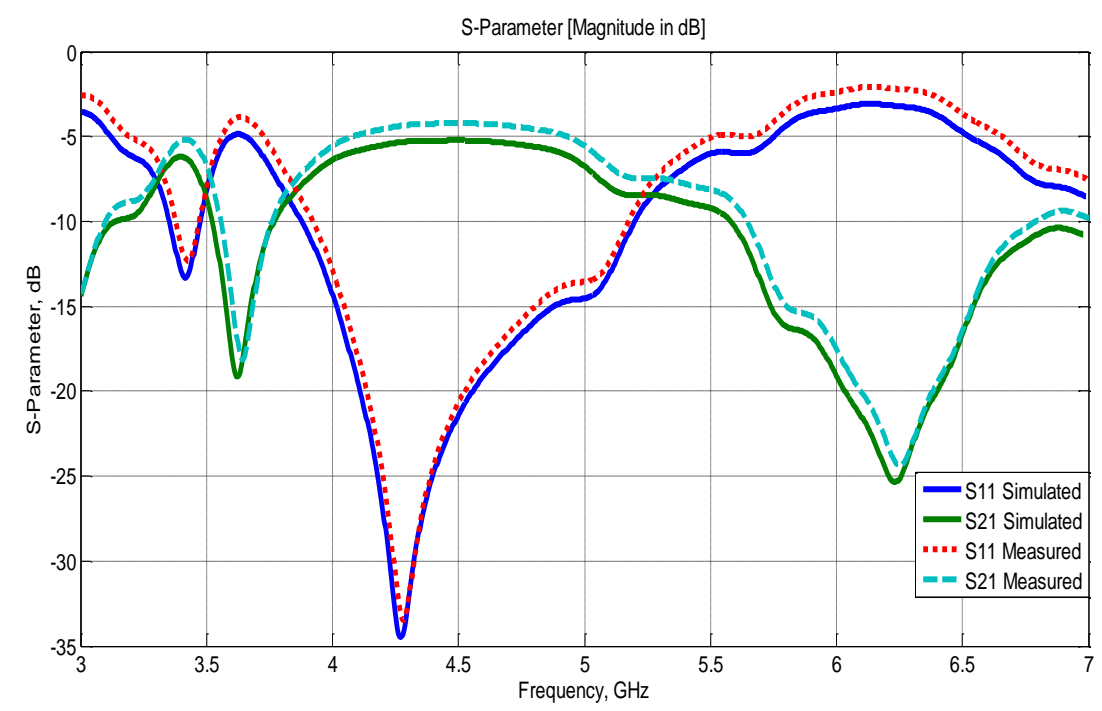

Figure 4. Simulated and Measured S11 and S21 Parameter

The simulated and measured $S_{11}$ and $S_{21}$ parameters are exported to MATLAB and using earlier equations of direct retrieval method, negative permeability and permittivity versus frequency are obtained as shown in Figures (5\&6) respectively. These figures give confirmation results of a negative behavior of the periodic structure, simulation and measured negative permeability and permittivity shows good agreement.

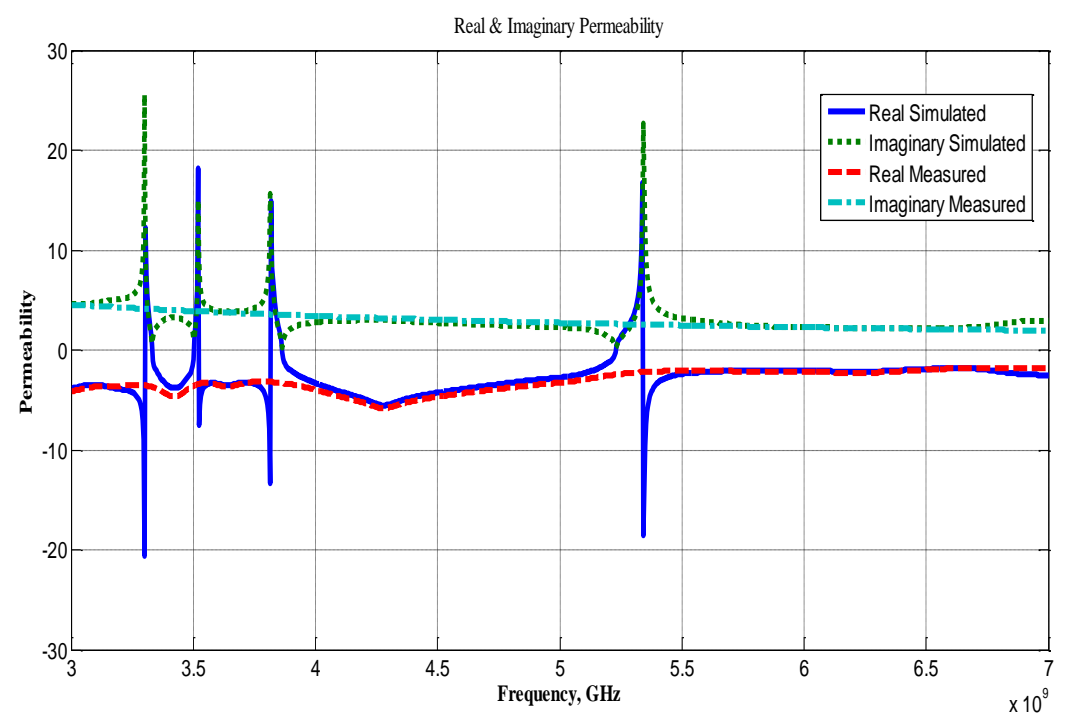

Figure 5. Simulated and Measured Permeability vs. Frequency, GHz 


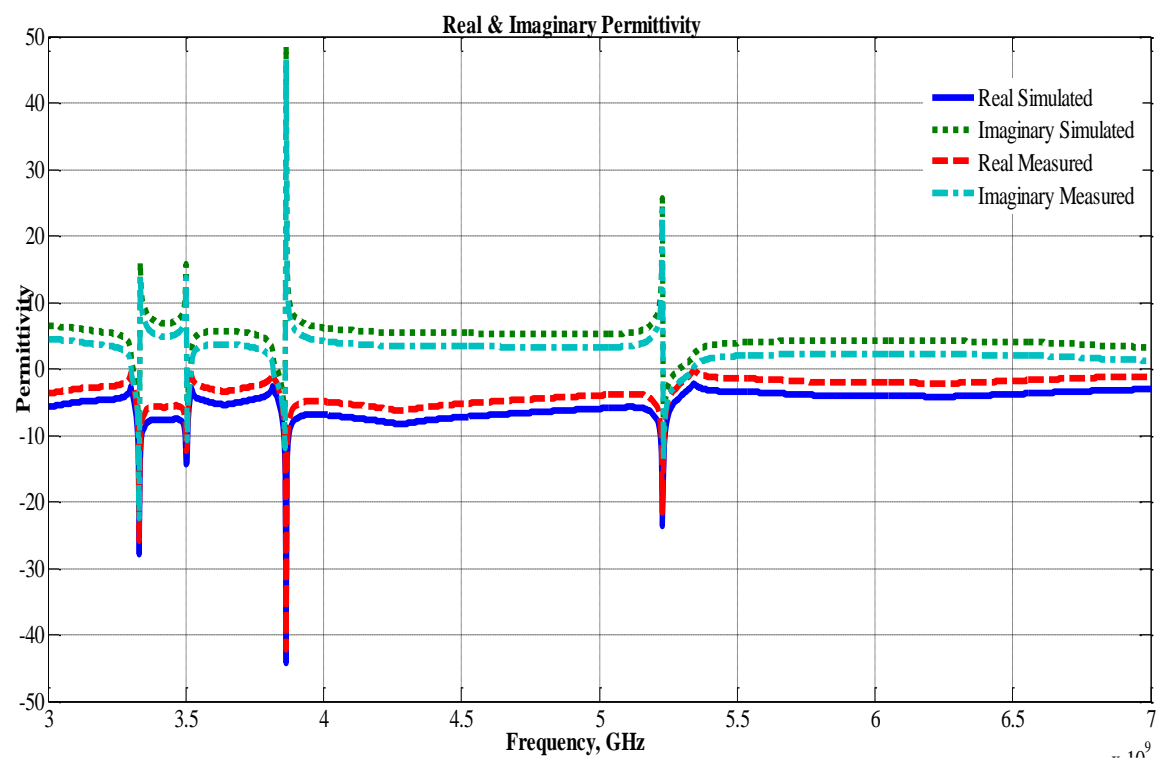

Figure 6. Simulated and Measured Permittivity vs. Frequency, GHz

Negative impedance and refractive index are also extracted from the MATLAB software using the same direct retrieval method as shown in Figures $(7 \& 8)$ respectively. Simulated and measured Negative impedance and refractive index shows good agreement. Over the given frequency range these Figs showed negative values of impedance and refractive index.

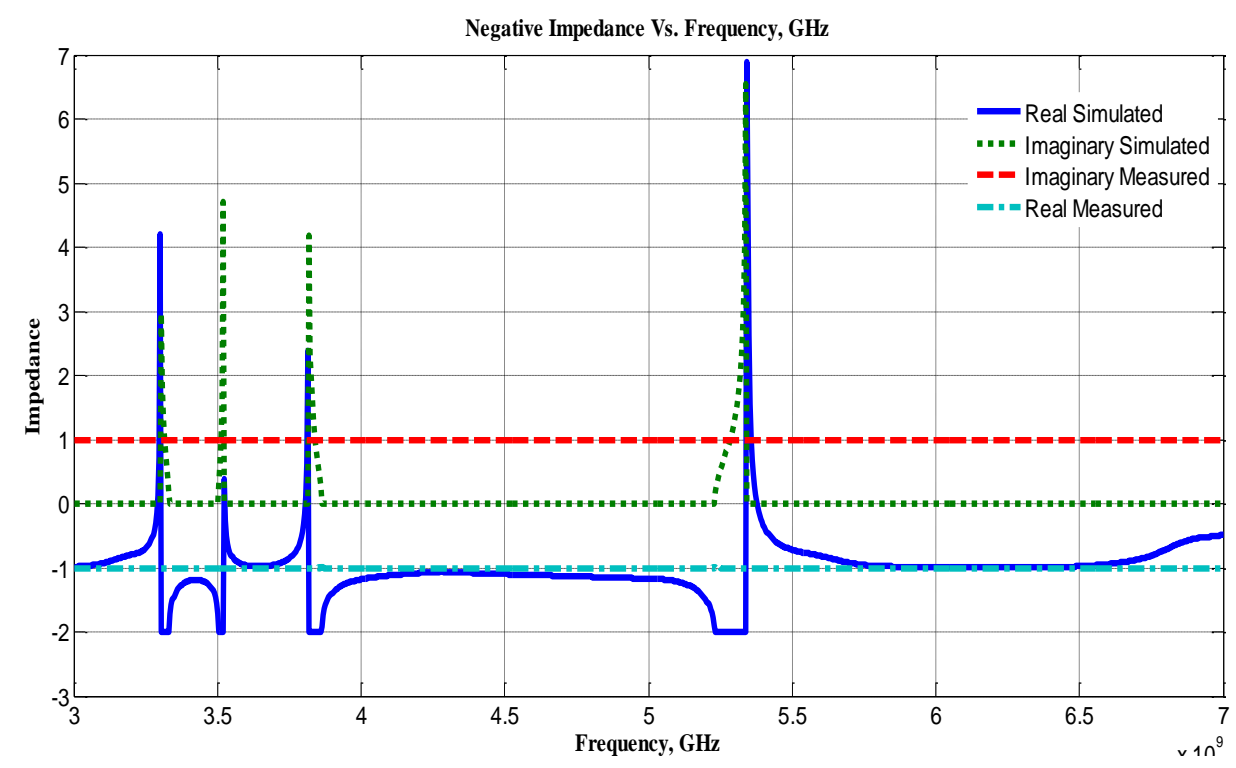

Figure 7. Negative Impedance vs. Frequency, GHz 


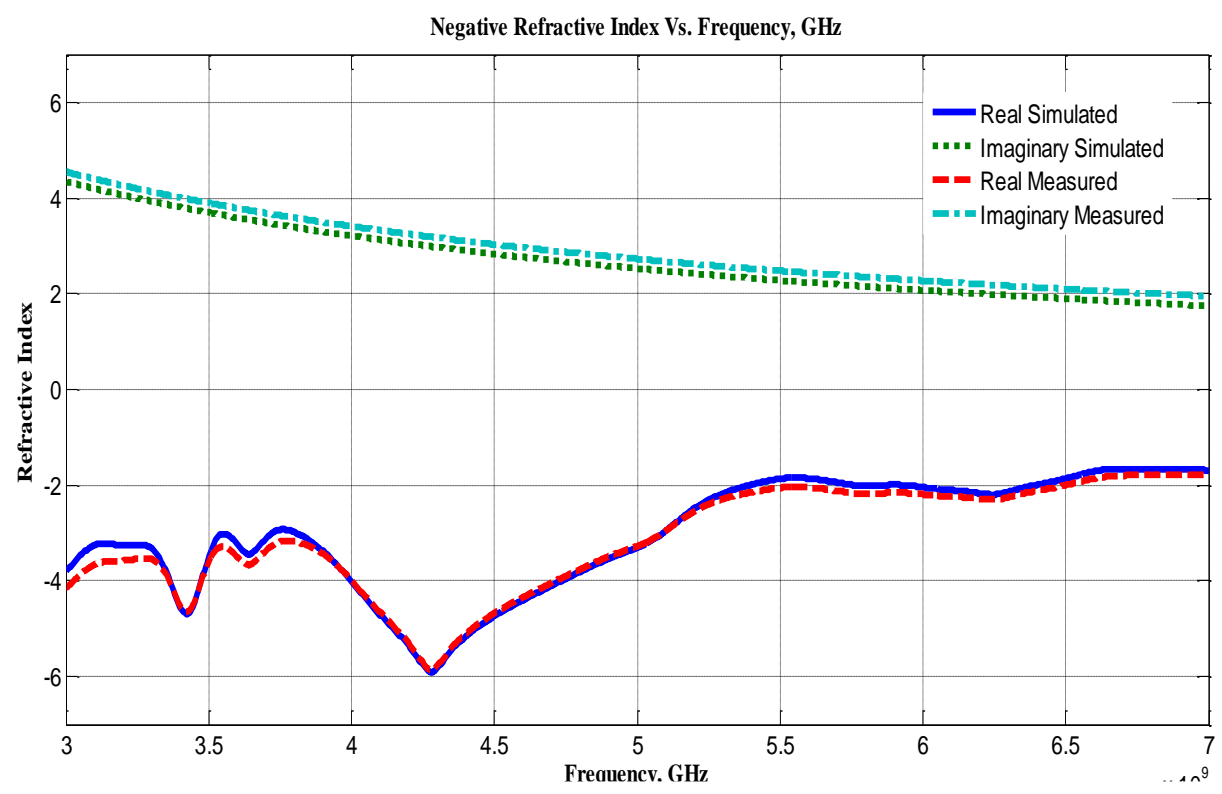

Figure 8. Negative Refractive Index Vs. Frequency, GHz

\section{Conclusion}

The designed and fabricated structures are successfully yields the negative values of permeability, permittivity, impedance, and refractive index. These parameters are produced using MATLAB from Transmission Reflection (T/R) method using direct retrieval technique from simulated and measured S11 and S21.The structure was fabricated using standard PCB fabrication. The experimental results well agree with the simulated ones. Negative parameters are obtained at different operating frequencies (3.8 to $5.2 \mathrm{GHz}$ ). Simulation and fabrication structure has simultaneous negative permeability and permittivity at the frequency range from $3.8 \mathrm{GHz}$ to $5.2 \mathrm{GHz}$ which gives left handed properties. The LHM structures in this work can interest, where LHM that have negative refractive index used as superlens to enhances gain and radiation characteristics of antennas at specific range of frequency. The structure can be widely used for enhancement of different antenna's parameters.

\section{References}

[1] V.G. Veselago, "The electrodynamics of substances with simultaneously negative values of $\varepsilon$ and $\mu$ ", Sov. Phys. Uspekhi, vol. 10, no. 4, (1968), pp. 509-514.

[2] H. Kosaka, T. Kawashima, A. Tomita, M. Notomi, T. Tamamura, T. Sato and S. Kawakami, "Superprism phenomena in photonic crystals", Phys. Rev. B, vol. 58, no. 16, (1998), pp. R10096R10099.

[3] J. B. Pendry, "Negative Refraction Makes a Perfect Lens", Phys. Rev. Lett., vol. 85, no. 18, (2000), pp. 3966-3969.

[4] J. B. Pendry, A. J. Holden, D. J. Robbins and W. J. Stewart, "Magnetism from conductors and enhanced nonlinear phenomena," IEEE Trans. Microw. Theory Tech., vol. 47, no. 11, pp. 2075-2084, 1999.

[5] D. R. Smith and N. Kroll, "Negative Refractive Index in Left-Handed Materials", Phys. Rev. Lett., vol. 85, no. 14, (2000), pp. 2933-2936.

[6] R. A. Shelby, D. R. Smith, S. C. Nemat-Nasser and S. Schultz, "Microwave transmission through a twodimensional, isotropic, left-handed metamaterial", Appl. Phys. Lett., vol. 78, no. 4, (2001), pp. 489-491.

[7] A. Grbic and G. V. Eleftheriades, "Experimental verification of backward-wave radiation from a negative refractive index metamaterial", J. Appl. Phys., vol. 92, no. 10, (2002), pp. 5930-5935.

[8] R. W. Ziolkowski, "Superluminal transmission of information through an electromagnetic metamaterial", Phys. Rev. E, vol. 63, no. 4, (2001), pp. 46604.

[9] J. B. Pendry, A. J. Holden, W. J. Stewart and I. Youngs, "Extremely Low Frequency Plasmons in Metallic Mesostructures”, Phys. Rev. Lett., vol. 76, no. 25, (1996), pp. 4773-4776. 
[10] D. R. Smith, W. J. Padilla, D. C. Vier, S. C. Nemat-Nasser and S. Schultz, "Composite Medium with Simultaneously Negative Permeability and Permittivity”, Phys. Rev. Lett., vol. 84, no. 18, (2000), pp. 4184-4187.

[11] R. A. Shelby, D. R. Smith and S. Schultz, "Experimental Verification of a Negative Index of Refraction", Science, vol. 292, no. 5514, (2001), pp. 77-79.

[12] D. R. Smith, D. C. Vier, T. Koschny and C. M. Soukoulis, "Electromagnetic parameter retrieval from inhomogeneous metamaterials", Phys. Rev. E, vol. 71, no. 3, (2005), pp. 36617.

[13] H. Zhou, "An Improved Method of Determining Permittivity and Permeability by S Parameters", PIERS Process. Beijing, vol. 11, no. 1, (2009), pp. 768-773.

[14] A. Mallik, S. Kundu and M. O. Goni, "Design of a novel two-rectangular U-shaped double negative metamaterial", in 2013 International Conference on Informatics, Electronics Vision (ICIEV), (2013), pp. $1-6$.

[15] M. Lapine and S. Tretyakov, "Contemporary notes on metamaterials", Antennas Propag. IET Microw., vol. 1, no. 1, (2007), pp. 3-11.

[16] H. Attia, L. Yousefi, M. M. Bait-Suwailam, M. S. Boybay and O. M. Ramahi, "Enhanced-Gain Microstrip Antenna Using Engineered Magnetic Superstrates”, IEEE Antennas Wirel. Propag. Lett., vol. 8, (2009), pp. 1198-1201.

[17] D. Jin, B. Li and J. Hong, "Gain improvement of a microstrip patch antenna using metamaterial superstrate with the zero refractive index", in 2012 International Conference on Microwave and Millimeter Wave Technology (ICMMT), (2012), vol. 3, pp. 1-3.

[18] B. Garg and P. k Singhal, "Design and Characterization of Compact Microstrip Patch Antenna Using 'Split Ring' Shaped Metamaterial Structure”, Int. J. Electr. Comput. Eng. IJECE, vol. 2, no. 5, (2012), pp. 655-662.

[19] A. N. Vicente, G. M. Dip and C. Junqueira, "The step by step development of NRW method", in Microwave Optoelectronics Conference (IMOC), 2011 SBMO/IEEE MTT-S International, (2011), pp. $738-742$.

[20] A. L. de Paula, M. C. Rezende and J. J. Barroso, "Modified Nicolson-Ross-Weir (NRW) method to retrieve the constitutive parameters of low-loss materials", in Microwave Optoelectronics Conference (IMOC), 2011 SBMO/IEEE MTT-S International, (2011), pp. 488-492.

[21] O. Luukkonen, S. I. Maslovski and S. A. Tretyakov, "A Stepwise Nicolson-Weir-Based Material Parameter Extraction Method”, IEEE Antennas Wirel. Propag. Lett., vol. 10, (2011), pp. 1295-1298.

[22] R. W. Ziolkowski, "Design, fabrication, and testing of double negative metamaterials", IEEE Trans. Antennas Propag., vol. 51, no. 7, (2003), pp. 1516-1529.

[23] N. Engheta and R. W. Ziolkowski, Metamaterials: Physics and Engineering Explorations. John Wiley \& Sons, (2006). 